\title{
El COVID-19, las élites y el futuro de la economía política de la reducción de la desigualdad en América Latina
}

\author{
Benedicte Bull y Francisco Robles Rivera
}

\section{Resumen}

Existen diversas opiniones en la literatura sobre cómo repercuten las pandemias en la desigualdad de los ingresos. Mientras que en la literatura económica se sostiene que las pandemias aumentan la desigualdad, la literatura histórica y de las ciencias políticas señala que las pandemias podrían ocasionar quiebres institucionales y, en un contexto de cambios a nivel de las élites y de presiones desde los estratos más bajos, mantener las desigualdades. Tras examinar los datos actuales sobre las repercusiones de la enfermedad por coronavirus (COVID-19), hemos constatado que se están produciendo transferencias de los ingresos hacia las clases más altas y determinados cambios a nivel de las élites en la región. Sin embargo, dado que las élites han controlado las medidas económicas que se han adoptado para mitigar y enfrentar la crisis, hay pocos indicios de que se esté produciendo un quiebre institucional.

\section{Palabras clave}

COVID-19, virus, epidemias, aspectos económicos, distribución del ingreso, élite, riqueza, pobreza, política económica, América Latina

\section{Clasificación JEL}

N46, P16, D36

\section{Autores}

Benedicte Bull es Profesora en el Centro para el Desarrollo y el Medio Ambiente (SUM) de la Universidad de Oslo (Noruega). Correo electrónico: benedicte.bull@sum.uio.no.

Francisco Robles Rivera es Investigador en el Instituto de Investigación Social y Profesor en la Escuela de Comunicación de la Universidad de Costa Rica. Correo electrónico: francisco.robles@ucr.ac.cr. 


\section{Introducción}

El debate acerca de los efectos de la enfermedad por coronavirus (COVID-19) sobre la desigualdad ha cambiado radicalmente en los últimos meses. Pese a que, en las primeras etapas de la propagación de la infección y las medidas de confinamiento, se describía al COVID-19 como "el gran igualador" (Mein, 2020), actualmente abundan las pruebas de que la pandemia aumentará la pobreza y la desigualdad (Busso y Messina, 2020). La Comisión Económica para América Latina y el Caribe (CEPAL) informa que, en algunos países, la pandemia podría ocasionar una variación de más de 3 puntos porcentuales en el índice de Gini, que mide la desigualdad, en tanto que el número de personas que viven en la pobreza aumentará un 4,4\% (28,7 millones más de personas) y se situará en el 34,7\% de la población de la región (CEPAL, 2020a). Esta situación obedece a diversos factores; entre ellos, a la pérdida generalizada de empleos mal remunerados y al hecho de que los trabajadores independientes del sector informal se han visto impedidos de realizar sus actividades económicas normales. Como contrapartida, los datos recientes indican que, desde marzo de 2020, los ingresos de las personas mejor remuneradas han aumentado (Ruiz, 2020).

La literatura acerca del impacto de las pandemias sobre la desigualdad no es concluyente. Algunos estudios demuestran que las pandemias afectan de manera desproporcionada a los pobres y que producen un aumento general en la desigualdad de los ingresos (Cohn y Alfani, 2007; Karlsson, Nilsson y Pichler, 2014). Otros señalan que las pandemias y otras crisis han tenido un efecto disruptivo que ha redundado en una redistribución de los activos y propiciado los cambios institucionales necesarios para promover la redistribución de los ingresos (Piketty, 2014; Scheidel, 2018). En este trabajo, se analiza el argumento de que, a la larga, el resultado dependerá de la medida en que las crisis modifiquen los recursos de las élites ${ }^{1}$ y su influencia sobre las instituciones y las políticas (Solimano, 2015). Si una crisis produce una transferencia de los ingresos hacia los estratos más altos de la sociedad sin provocar ningún cambio en las élites, cabe esperar una mayor desigualdad. Si, por el contrario, la crisis obliga a las élites a prestar más atención a las exigencias de los grupos desfavorecidos, es posible que se suscite una reestructuración de las instituciones que perpetúan la cultura de privilegio y que, a largo plazo, la desigualdad se reduzca. No obstante, también es posible que las élites que actualmente están sufriendo pérdidas a raíz de la pandemia busquen aferrarse a sus privilegios ejerciendo un control más férreo sobre las instituciones y oponiendo resistencia a las medidas de redistribución.

En este trabajo, examinamos los escasos datos disponibles sobre la concentración de ingresos desde el comienzo de la pandemia del COVID-19 entre quienes tienen las mayores remuneraciones, así como la información sobre la distribución de los recursos entre los diferentes grupos de élite y sus reacciones a las medidas impulsadas para controlar la pandemia. Sobre esta base, buscaremos determinar si el COVID-19 podría tener un efecto disruptivo que propicie una mejor distribución de los recursos, o si simplemente producirá una transferencia de los recursos hacia las clases más altas, sin generar cambios en el statu quo.

El trabajo se divide en siete secciones, incluida la presente introducción. En la sección II se examina la literatura sobre la relación entre las pandemias y la desigualdad, en tanto que en la sección III se presenta una breve reseña de la desigualdad económica en América Latina. En la sección IV se analiza la transferencia de ingresos hacia las clases más altas durante la pandemia del COVID-19, y en la V se examina la cuestión de si la crisis está generando cambios a nivel de las élites. La sección VI se aboca a la influencia de las élites sobre las medidas gubernamentales para enfrentar la pandemia, y la sección VII concluye este trabajo.

\footnotetext{
1 El término "élite" se refiere a los grupos de personas cuyo control sobre los recursos naturales, económicos, políticos, coercitivos, sociales, organizacionales o simbólicos (a saber, sus habilidades o conocimientos) los coloca en una posición que les permite ejercer influencia formal o informal sobre las organizaciones y las prácticas institucionales (Bull, 2014).
} 


\section{Las pandemias, la desigualdad y las élites}

Existen datos concluyentes que evidencian que la desigualdad ha sido uno de los principales factores que han determinado el alcance y las repercusiones de las pandemias a lo largo de la historia. Durante la pandemia de la gripe española de 1918, se registraron importantes variaciones en las tasas de mortalidad en función de los ingresos dentro de los países y entre ellos, en particular en las ciudades y los pueblos con tasas elevadas de desigualdad social (Mamelund, 2017). Durante la pandemia de la gripe A (H1N1), en 2009, la tasa de mortalidad fue 20 veces más elevada en algunos países de América del Sur que en Europa, y tres veces superior en los quintiles socioeconómicos más desfavorecidos de la población de Inglaterra que en el quintil más acaudalado (Rutter y otros, 2012; Simonsen y otros, 2013). En su análisis estadístico, Peter Turchin determinó que los niveles de desigualdad y de interconexión mundial y los efectos de las pandemias guardan una estrecha relación (Turchin, 2018). También existen cada vez más pruebas de que las tasas de contagio y de letalidad del COVID-19 son más altas entre los grupos de personas pobres y marginadas (Galasso, 2020). Si bien, en las anteriores pandemias, la principal causa de las mayores tasas de infección y de letalidad entre los pobres fue el deterioro de las condiciones sanitarias, de salud y de vivienda, las actuales desigualdades en materia digital y laboral también dificultan el cumplimiento de las medidas de distanciamiento físico para algunos grupos.

Sin embargo, las pruebas no son tan concluyentes en lo referido a la forma en que las pandemias afectan los patrones de desigualdad. La literatura económica concluye que las pandemias y las epidemias generalizadas han aumentado la desigualdad. En su estudio de cinco eventos de este tipo acaecidos desde 2000, Furceri y otros (2020) concluyeron que las pandemias generan un persistente aumento de la desigualdad medida según el coeficiente de Gini. Su modelo demuestra que, cinco años después de una pandemia, los coeficientes de Gini de los ingresos de mercado y de los ingresos netos aumentan un $0,75 \%$ y un $1,25 \%$, respectivamente. Las pandemias también aumentaron la participación de los deciles más altos en los ingresos (Furceri y otros, 2020). Lo anterior es congruente con otras pruebas que señalan que los acontecimientos traumáticos, como las guerras o las crisis financieras, en general aumentan la desigualdad (De Haan y Sturn, 2017). Entre otras cosas, esto obedece al hecho de que las personas con mayores ingresos están mejor preparadas para adaptarse a los cambios en los mercados laborales y aprovechar las nuevas oportunidades de negocios.

En la literatura histórica y de las ciencias políticas, se llega a otras conclusiones. Las pandemias han estado entre los mayores igualadores de la riqueza y de los ingresos de la historia, junto con las campañas bélicas, las revoluciones y el derrumbe de los Estados (Scheidel, 2018). Por ejemplo, las redistribuciones ocurridas a mediados del siglo XX en Europa y en los Estados Unidos pueden entenderse como el resultado de una sucesión de crisis mundiales: la Primera Guerra Mundial, la Segunda Guerra Mundial y la Gran Depresión (Piketty, 2014; Starr, 2019). Uno de los motivos por el que las pandemias favorecen la distribución de los ingresos es que tienen mayor incidencia sobre los pobres, producen escasez de mano de obra y crean condiciones para que se produzca un aumento de los salarios. Durante los períodos de estabilidad y crecimiento económicos, las élites procuran establecer leyes y normas que promuevan la concentración y la transferencia intergeneracional de la riqueza (Fukuyama, 2016; Scheidel, 2018). Pueden hacerlo gracias a su capacidad de invertir y generar empleo (poder estructural) y de impulsar acciones políticas (poder instrumental) (Fairfield, 2015). En América Latina, la cultura de privilegio que se desarrolló a lo largo del tiempo es un sistema de valores que perpetúa las ventajas de determinados grupos de la población en función de su posición socioeconómica, su condición de miembros de élites políticas y culturales o su ascendencia (Bielschowsky y Torres, 2018). En contraste, en épocas de crisis, la prioridad es la supervivencia a corto plazo y la superación de amenazas. Es en estos momentos cuando los ideales de la igualdad social ganan aceptación, por cuanto las élites se ven presionadas a cooperar con las clases más bajas y mejorar su participación en los ingresos, 
lo que tiene efectos de largo plazo sobre la distribución de la riqueza (Peacock y Wiseman, 1961; Starr, 2019)². Si bien la disminución de la desigualdad de ingresos también podría ser la consecuencia de los períodos de estabilidad y crecimiento - como sucedió en América Latina entre 2003 y 2014 sin distribución de los ingresos y cambios institucionales, tales episodios podrían ser de corto aliento (Rodríguez Weber, 2018).

Con todo, los resultados de las pandemias y de otras crisis no siempre son los mismos. Collier y Collier (1991) demuestran que la incorporación de representantes de los trabajadores a las instituciones políticas durante las "coyunturas críticas" de la década de 1930 conformó las trayectorias de la democracia y condujo a resultados muy distintos en los años que siguieron. De la misma forma, la manera en que las instituciones se enfrenten al COVID-19 afectará el futuro de la desigualdad y de la democracia. Las investigaciones no respaldan la idea generalizada de que la desigualdad conduce al fracaso de la democracia ${ }^{3}$. No obstante, los niveles elevados de concentración de los ingresos sí afectan la calidad de la democracia, y en particular, la capacidad de las instituciones democráticas de promulgar, aplicar y sostener las reformas distributivas, por cuanto las élites poderosas disponen de más recursos para oponerse a las políticas de redistribución (Acemoglu y Robinson, 2020; Moene, 2015). En parte, esto se debe a que cuanto más concentrados estén la riqueza y los recursos, más débiles serán determinados actores democráticos fundamentales, como los medios de comunicación, los movimientos sociales y los sindicatos (Durand, 2019).

Las conclusiones de ambos tipos de literatura en lo referido a los efectos que las pandemias tienen sobre la desigualdad no necesariamente son contradictorias. El aumento de la desigualdad que se describe en la literatura económica, que se centra en períodos limitados de tiempo, responde a las observaciones de los efectos directos de las pandemias y otras crisis. La conclusión que ofrece la literatura histórica, a saber, que el resultado a largo plazo de una crisis depende de que se introduzcan cambios en las fuentes de poder de las élites y de que estas presten apoyo a las instituciones y a las medidas de redistribución, implica que una transferencia de los ingresos hacia los niveles más altos de la sociedad sin ejercer presión para suscitar una disrupción institucional profundizará la desigualdad. Sin embargo, si la crisis genera una redistribución de los ingresos y crea presiones desde las bases, es más probable que las élites celebren nuevos pactos sociales que reduzcan la desigualdad a largo plazo. La persistencia de las condiciones estructurales que hacen de América Latina la región más desigual del mundo a menudo obedece a la capacidad de las élites de preservar las instituciones generadoras de desigualdad, aun durante los períodos democráticos caracterizados por grandes movilizaciones sociales (Rovira-Kaltwasser, 2018).

\section{El punto de comienzo: la concentración de la riqueza y la inercia estructural en América Latina}

La reducción de la pobreza y la desigualdad en América Latina entre 2003 y 2014 puede atribuirse a una combinación entre las condiciones favorables de la economía mundial, las mejoras en los niveles educativos (lo que redujo las diferencias salariales entre los trabajadores calificados y

\footnotetext{
2 Fue eso lo que sucedió en el caso de los conflictos sociales de la década de 1930 en los países escandinavos, que llevaron a la firma de una serie de acuerdos entre los trabajadores y los empleadores que, durante los siguientes siete decenios, permitieron disminuir la desigualdad en los ingresos (Bull, 2019).

3 Las afirmaciones de que la democracia conduce a una distribución equitativa de la riqueza y de que la desigualdad en el reparto de los recursos conlleva el fracaso de la democracia tienen escasos fundamentos. Asimismo, la idea generalizada de que América Latina siempre ha sido más desigual que otras regiones y, por ende, menos democrática, también ha sido rebatida desde varios frentes. Véase Scheve y Stasavage (2017).
} 
los no calificados) y las políticas de mercado sensibles a las cuestiones sociales y laborales (Busso y Messina, 2020) ${ }^{4}$.

Sin embargo, la reducción de la desigualdad en América Latina prácticamente no tuvo efectos sobre los ingresos de quienes ganan los salarios más altos. Los escasos datos disponibles indican que el $1 \%$ mejor remunerado recibe alrededor del $20 \%$ del total de los ingresos, y hay pocos indicios de que ese porcentaje haya disminuido (Alvaredo y otros, 2018). Nuevos datos en Morgan (2017) demostraron que en 2015 el 1\% más rico en el Brasil captó un 28,3\% del total de los ingresos, y que el $10 \%$ mejor remunerado recibió más de la mitad de ese total, a saber, un $55,6 \%{ }^{5}$. En lo referido a Chile, los datos sobre el período transcurrido entre 2005 y 2010 muestran que el 1\% con los mejores salarios recibió alrededor del 30,5\% del total de ingresos, y que un 10,1\% de ese total quedó en manos del 0,01\% más rico (López Vega, Figueroa y Gutiérrez, 2013). Las estimaciones del Banco Mundial (2018b) señalan que, en promedio, el 10\% más rico de la región recibe una tercera parte de los ingresos nacionales (véase el cuadro 1).

\section{Cuadro 1}

América Latina (17 países): porcentaje de los ingresos nacionales en manos del 10\% más rico e índice de Gini, por país, 2008 a 2018

(En porcentajes, valores medios para el período)

\begin{tabular}{lcc}
\hline & Ingresos en manos del $10 \%$ más rico & Índice de Gini \\
\hline Brasil & 41,95 & 53,14 \\
\hline Colombia & 41,45 & 52,30 \\
\hline Honduras & 39,97 & 52,57 \\
\hline Panamá & 39,06 & 51,03 \\
\hline Paraguay & 38,55 & 49,07 \\
\hline Guatemala & 38,00 & 48,30 \\
\hline México & 37,98 & 47,70 \\
\hline Costa Rica & 37,27 & 48,73 \\
\hline Chile & 37,06 & 45,52 \\
\hline República Dominicana & 36,07 & 46,08 \\
\hline Nicaragua & 35,70 & 45,05 \\
\hline Ecuador & 35,50 & 46,52 \\
\hline Bolivia (Estado Plurinacional de) & 34,30 & 46,46 \\
\hline Perú & 33,28 & 44,48 \\
\hline El Salvador & 32,55 & 42,04 \\
\hline Uruguay & 31,06 & 41,50 \\
\hline Argentina & 30,58 & 42,53 \\
\hline Fuente: Elabora
\end{tabular}

Fuente: Elaboración propia, sobre la base de Banco Mundial, "Índice de Gini”, 2018 [en línea] https://datos.bancomundial.org/indicador/SI.POV.GINI y "Participación en el ingreso del 10\% mejor remunerado de la población”, 2018 [en línea] https://datos.bancomundial.org/ indicador/SI.DST.10TH.10.

Nota: Los datos de Guatemala corresponden a 2014. Los datos de Nicaragua corresponden a 2009 y a 2014.

Además, no se produjo ninguna redistribución importante de los activos (Cornia, 2014). En América Latina, el coeficiente de Gini de la riqueza es mucho más alto que el coeficiente promedio referido a los ingresos, si bien existen pocos datos históricos comparables (Amarante y Jiménez, 2015). Con todo, hay pocos indicios de que los niveles de desigualdad en lo concerniente a los activos hayan disminuido, o de que se haya producido algún otro cambio estructural.

\footnotetext{
4 La principal excepción a esta tendencia fue Costa Rica, donde se produjo un aumento de la desigualdad (Trejos y Oviedo, 2012).

5 Las cifras de ingresos corresponden al ingreso nacional bruto.
} 
Ante la ausencia de cambios estructurales que modifiquen los patrones de distribución de los activos y transformen a las élites que los controlan, los episodios de reducción de la desigualdad podrían ser de corta duración. Lejos de embarcarse en un proyecto de redistribución profunda de la riqueza, los sucesivos gobiernos del Partido de los Trabajadores (PT) en el Brasil impulsaron una agenda favorable a los pobres y una estrategia de crecimiento basada en la igualdad que, en última instancia, hizo que el proceso fuese insostenible (Loureiro, 2020). En Colombia, existen dos factores principales que han impedido mejorar la distribución de los ingresos: en primer lugar, las élites propietarias de la tierra históricamente han moldeado en su beneficio las políticas y las decisiones locales (Faguet, Sánchez y Villaveces, 2020); y, en segundo lugar, existen poderosos grupos económicos que influyen en las políticas fiscales con el objetivo beneficiarse de los sistemas de tributación indirecta y evitar el aumento de los impuestos a los ingresos (Castañeda, 2018). Honduras es un caso particular, ya que la suma debilidad de las instituciones democráticas ha hecho que los grupos de élite se valgan del control que ejercen sobre el dinero, el poder y los medios, lo que, a su vez, ha socavado los intentos de impulsar cualquier política de redistribución (Euraque, 2019; Sosa Iglesias, 2017). En Panamá, el nuevo marco constitucional creado en 1999 brindó incentivos para que las élites empresariales promovieran y desarrollaran un régimen liberal ventajoso en el país (Kasahara, 2012; Pérez, 2011). No obstante, desde 2009, los conflictos internos entre las élites para hacerse con el control del gobierno y los escándalos de corrupción han dejado claro que dentro de la élite empresarial existe una facción pequeña, pero poderosa, que puede ejercer un considerable control directo sobre la administración del Estado para promover sus propios intereses (Cárdenas y Robles Rivera, 2020; Santos y Fraga, 2020). Ahora la principal cuestión es cómo la crisis del COVID-19 afectará el equilibrio de poder entre los intereses de las élites.

\section{Transferencias de ingresos a las clases más altas durante la pandemia del COVID-19}

Los datos recientes parecen indicar que, durante la pandemia de COVID-19, los ingresos y las fortunas de las personas mejor remuneradas y de los dueños de la riqueza han aumentado, lo que va en contra de las estimaciones iniciales. Aunque no disponemos de datos comparables para el 1\% más rico ni para el 10\% mejor remunerado desde el inicio de la pandemia, hemos usado los datos sobre los ingresos y la riqueza de las personas mejor remuneradas que surgen, entre otras fuentes, de los estados financieros de las empresas más importantes ${ }^{6}$.

Con base en esos datos, Gneiting, Luisani y Tamir (2020) llegaron a la conclusión de que, a nivel mundial, el pago excesivo de dividendos antes del inicio de la pandemia de COVID-19 es uno de los principales motivos de los problemas financieros que actualmente enfrentan varias empresas importantes. Entre 2010 y 2019, las empresas que conforman el índice S\&P 500 gastaron 9,1 billones de dólares de los Estados Unidos, equivalentes a más del 90\% de sus ganancias durante el mismo período, en pagos de dividendos a sus acaudalados accionistas. Desde enero de 2020, muchas empresas importantes han aumentado sus pagos a los accionistas. Por ejemplo, los estados financieros de Microsoft y Google indican que, desde enero, estas empresas han pagado a sus accionistas más de 21.000 millones y 15.000 millones de dólares, respectivamente. Esto no ocurre solo en las empresas tecnológicas. Entre enero y julio de 2020, las seis empresas petroleras más importantes del mundo 7 sufrieron pérdidas netas del orden de los 61.700 millones de dólares de los Estados Unidos, pero

\footnotetext{
6 Gneiting, Luisani y Tamir (2020) recurrieron a la plataforma S\&P Capital IQ, una base de datos de análisis financieros dirigida a los inversores, y en la medida necesaria, corroboraron la información analizando cuidadosamente los estados financieros anuales y trimestrales de las empresas.

7 Las empresas son Exxon Mobil, Total, Shell, Petrobras, Chevron y BP. Véase Coffey y otros (2020).
} 
igualmente se las arreglaron para pagar 31.000 millones de dólares a sus accionistas durante el mismo período (Gneiting, Lusiani y Tamir, 2020).

En un análisis de las tendencias en América Latina referidas a la variación del patrimonio neto de los multimillonarios de la región entre el 18 de marzo y el 12 de julio de 2020, Oxfam (2020) constató que, durante ese período, el valor patrimonial neto combinado de los multimillonarios de la Argentina pasó de 8.800 millones a 11.200 millones de dólares de los Estados Unidos; en el Brasil, de 123.100 millones a 157.100 millones; en Colombia, de 13.700 millones 14.100 millones; en Chile, de 21.000 millones a 26.700 millones; en el Perú, de 5.200 millones a 5.500 millones, y en la República Bolivariana de Venezuela, de 3.400 millones a 3.500 millones de dólares ${ }^{8}$.

En total, la riqueza de los 73 latinoamericanos más ricos aumentó en 48.200 de dólares de los Estados Unidos; es decir, un 17\% desde el comienzo de la pandemia. Desde el comienzo de las medidas de confinamiento, cada dos semanas hay un nuevo multimillonario en la región (Ruiz, 2020). Todavía es muy prematuro decir cómo afectará la pandemia a la categoría compuesta por los individuos de alto patrimonio neto ${ }^{9}$, que en cierto modo es más amplia. El Informe Mundial de la Riqueza 2020 demuestra que, si bien el crecimiento de la riqueza mundial se estancó en el período 2018-2019, el número de personas en esa categoría aumentó un 8,6\%. En los Estados Unidos, el aumento fue del orden del $11 \%$, un incremento sin precedentes. El número de individuos de alto patrimonio neto en América Latina también aumentó durante este período de escaso crecimiento, si bien apenas un 2,7\%. El informe destaca que, pese a la desaceleración de la economía mundial debido al COVID-19, los mercados financieros podrían mostrarse resilientes y ofrecer buenas perspectivas mundiales para los individuos de este grupo (Capgemini, 2020). Por ende, si bien la recuperación de los mercados de capital de América Latina ha sido lenta y desigual desde que se desplomaron, en el período marzo-abril de 2020, el efecto sobre los ingresos más altos podría no ser grave.

Al mismo tiempo, los datos de junio de 2020 de la Organización Internacional del Trabajo (OIT) indican que el drástico aumento del desempleo a raíz del COVID-19 dejó desempleados a 41 millones de latinoamericanos, una cifra sin precedentes (OIT, 2020a). Si bien, en crisis anteriores, el desempleo en América Latina ha afectado a los miembros de las clases medias, el desempleo originado por el COVID-19 ha tenido especial incidencia en los pobres debido a la naturaleza de su trabajo (Busso y Messina, 2020). Del 40\% de los latinoamericanos más pobres, menos del 10\% puede trabajar desde su hogar, o bien porque no acceden a las tecnologías de la información y las comunicaciones, o bien porque sus empleos les exigen presencialidad física (Delaporte y Peña, 2020). Las primeras investigaciones procedentes de España y de los Estados Unidos que pueden aplicarse a América Latina dejan claras dos tendencias principales (Aspachs y otros, 2020; Béland, Brodeur y Wright, 2020). En primer lugar, se produjo un aumento de la tasa de desempleo y una disminución del horario laboral y la participación de la fuerza de trabajo. En segundo lugar, el impacto negativo fue mayor sobre los trabajadores jóvenes, los migrantes y los de menor nivel educativo. Ambas tendencias agravarán las desigualdades en el mercado laboral (Béland, Brodeur y Wright, 2020). Según estimaciones de la OIT, la pérdida de horas de trabajo en América Latina y el Caribe en el tercer trimestre de 2020, en relación con el cuarto trimestre de 2019 , fue de un $25,6 \%$. A esto se suma una marcada caída de los ingresos laborales en los primeros tres trimestres de 2020, con una retracción del 19,3\% como porcentaje de los ingresos laborales y de un 10,1\% como porcentaje del producto interno bruto (PIB) (OIT, 2020b).

El COVID-19 también ha tenido efectos negativos sobre las pequeñas y medianas empresas (pymes), y en particular sobre las microempresas. Los primeros datos de la CEPAL indicaban que un $7,1 \%$ de las pequeñas empresas y un $20,7 \%$ de las microempresas cerrarían antes de que terminase 2020 (CEPAL, 2020b). Datos posteriores de la Organización para la Cooperación y el Desarrollo

\footnotetext{
8 Véase también Ruiz (2020).

9 Se refiere a personas con activos invertibles por valor de 1 millón de dólares de los Estados Unidos o más, sin incluir la residencia principal, sumas por cobrar, bienes fungibles y bienes de consumo duraderos.
} 
Económicos (OCDE) parecen indicar que el número será mucho más alto. Durante la primera mitad de 2020, el 38\% de las pymes de América Latina cerraron, y un 27\% de ellas permanecían cerradas a finales de junio (OCDE/Facebook, 2020).

En crisis anteriores, la apertura de pymes funcionó como una suerte de "estrategia de supervivencia" (Mungaray Lagarda y otros, 2015). Asimismo, las pymes de América Latina siempre han sido consideradas estructuras sumamente adaptables a las circunstancias adversas, es decir, durante las crisis (Bernal y Michel, 2016). Sin embargo, las estrictas medidas de confinamiento y el desplome de la demanda de bienes y servicios han impedido a las pymes superar su mayor obstáculo en tiempos de crisis, a saber, sus niveles generalmente bajos de liquidez (OCDE/Facebook, 2020).

Si bien todavía es demasiado prematuro sacar conclusiones concretas, los datos mencionados sugieren que la riqueza se está transfiriendo hacia las clases más altas, de los segmentos de menores ingresos a los de altos ingresos y, especialmente, a quienes tienen las mejores remuneraciones y a los propietarios de la riqueza. Es probable que esto agrave la desigualdad. Sin embargo, también es posible que la riqueza se traslade de un grupo de élite a otro.

\section{V. ¿Producirá el COVID-19 cambios en la situación de las élites?}

La transferencia de la riqueza hacia las clases más acaudaladas no se ha producido de la misma manera en todos los sectores. La imposición de cuarentenas a millones de personas en toda la región, aunada al cierre de cientos de comercios, han beneficiado a las plataformas digitales. En la Argentina, las acciones de dos importantes empresas - Mercado Libre y Globant - han experimentado marcados aumentos de precios. En el caso de Mercado Libre, entre agosto de 2019 y agosto de 2020, el precio de las acciones pasó de 602 a 1.277 dólares (véase el cuadro 2), en tanto que sus ventas subieron un 45\% en comparación con las de 2019 (Ventrici, Krepki y Palermo, 2020). Por su parte, el precio de las acciones de Globant prácticamente se duplicó de un año al siguiente. Algunas de las empresas digitales más importantes del Brasil, como PagSeguro Digital y StoneCo Ltd, los precios de cuyas acciones vienen aumentando desde el comienzo de la pandemia, también tuvieron buenos resultados (Kitchener, 2020).

\section{Cuadro 2}

Argentina y Brasil: variación del precio de las acciones de las plataformas digitales, 2019 y 2020 (En dólares de los Estados Unidos)

\begin{tabular}{lccc}
\hline Empresa & $\begin{array}{c}\text { Precio por acción } \\
\text { el 26 de agosto de 2019 }\end{array}$ & $\begin{array}{c}\text { Precio por acción } \\
\text { el 23 de marzo de 2020 }\end{array}$ & $\begin{array}{c}\text { Precio por acción } \\
\text { el 27 de agosto de 2020 }\end{array}$ \\
\hline StoneCo Ltd (BRA) & 30,51 & 19,89 & 50,56 \\
\hline PagSeguro Digital Ltd (BRA) & 50,30 & 14,95 & 44,19 \\
\hline Mercado Libre (ARG) & 602,62 & 457,65 & 1201,40 \\
\hline Globant (ARG) & $92,89^{a}$ & 73,50 & 177,00 \\
\hline
\end{tabular}

Fuente: Elaboración propia, sobre la base de National Association of Securities Dealers Automated Quotations (NASDAQ), "Stocks" [en línea] https://www.nasdaq.com/market-activity/stocks, y Bolsa de Nueva York, "Listings Directory" [en línea] https://www.nyse.com/listings_directory/stock.

a Este valor corresponde al 28 de agosto de 2019.

Con la pandemia también ha aumentado la importancia de ciertas empresas para los gobiernos, y con ello, su potencial influencia. Desde el comienzo, el Gobierno de la Argentina viene cooperando estrechamente con gigantes tecnológicos de la talla de Amazon, Microsoft y Google, así como con 
Globant y Mercado Libre, para usar sus datos con el fin de controlar la propagación del virus a nivel nacional (Ventrici, Krepki y Palermo, 2020). Dos de estas iniciativas son un tablero digital para hacer un seguimiento de las camas y los respiradores disponibles en los hospitales del país, y una aplicación móvil de salud llamada Cuidar, que permite a los usuarios realizar autodiagnósticos, rastrear sus contactos y tramitar permisos de circulación. El tablero fue desarrollado en tiempo récord por Globant y la empresa estadounidense Salesforce, en tanto que la aplicación fue el fruto de un proyecto de múltiples asociados dirigido por la Secretaría de Innovación Pública. Sin embargo, el Gobierno depende por completo de las empresas asociadas para que la aplicación siga funcionando (Ventrici, Krepki y Palermo, 2020). De hecho, la creciente riqueza de las empresas tecnológicas, aunada a la dependencia de los gobiernos de las plataformas que ofrecen, reducirá las posibilidades de establecer el impuesto digital que la sociedad civil y los organismos internacionales han propuesto (CEPAL, 2019).

Gracias a la pandemia, la economía digital está floreciendo en toda la región, y algunos informes de prensa prevén que representará un 40\% del PIB regional para 2022 (Mari, 2019). Sin embargo, por su misma naturaleza, las economías digitales tienden a concentrar la riqueza en unas pocas empresas. Los primeros datos señalan que las ganadoras en esta pandemia son las empresas dedicadas a los ámbitos de la biotecnología, los productos farmacéuticos, la medicina y el comercio electrónico (Sokol y Pataccini, 2020).

Por otro lado, las medidas de confinamiento han reducido las exportaciones, el flujo de remesas y el turismo, tres fuentes clave de ingresos y riqueza en la región. En primer lugar, los datos correspondientes al primer trimestre de 2020 muestran que, en América Latina, el valor de las exportaciones se redujo un 3,2\%, en un contexto de contracción económica que presionó a la baja los precios del petróleo $(-32,0 \%)$ y del cobre $(-11,9 \%)$, así como los de la soja $(2,2 \%)$ y el café $(4,4 \%)$ (Giordano, 2020). En segundo lugar, el volumen de remesas ha disminuido. Los datos preliminares señalan que, en abril de 2020, las remesas fueron un $17 \%$ más bajas que en el mismo mes del año anterior en países que dependen de ellas en grado sumo, como Colombia, El Salvador, Guatemala, Honduras, México y la República Dominicana (Noe-Bustamante, 2020). Las repercusiones sobre la relación entre las élites podrían ser profundas en países como Honduras y El Salvador, donde los grupos económicos han tenido que adaptar sus estrategias para captar las remesas, que en 2019 representaron alrededor del 20\% del PIB (Bull, Castellacci y Kasahara, 2014; Rocha, 2008). En tercer lugar, el desplome del turismo podría traer aparejada una caída de 8 puntos porcentuales del PIB en el Caribe y de 1 punto porcentual en América Latina (CEPAL, 2020c). Esto debilitará a las élites del sector de los servicios.

Aunque de una naturaleza completamente distinta, otro cambio de situación a nivel de las élites podría vincularse con el fortalecimiento de las élites militares gracias a su papel como responsables de mantener el control de la población durante las medidas de confinamiento. Tal cambio ya ha ocurrido en países como Nicaragua y El Salvador, así como en Bolivia (Estado Plurinacional de), Chile, Colombia y Venezuela (República Bolivariana de). En algunos casos, esto podría aumentar el control del ejército sobre la economía. Por ejemplo, en la República Bolivariana de Venezuela, se ha producido un aumento en las extorsiones en el marco de la pandemia de COVID-19 (De Jesús, 2020). Los actos de extorsión, tanto los cometidos por bandas como por grupos con conexiones militares, en ocasiones han llevado a que grupos armados se hagan con el control de pequeñas empresas (Bull, 2020). Por lo tanto, es posible que el papel de las élites militares en la economía siga fortaleciéndose.

En suma, la pandemia podría ocasionar cambios en el control de los recursos. Los grupos de élite en los sectores tecnológico, farmacéutico, de la atención de la salud y de la economía digital obtendrán beneficios, en tanto que los propietarios de los centros comerciales o quienes dependen de las exportaciones, las remesas y el turismo - sectores que tradicionalmente percibían los mayores ingresos - quizás ahora necesiten el apoyo del gobierno para seguir funcionando. 


\section{Reacciones de las élites y de los gobiernos ante las iniciativas de redistribución durante la pandemia de COVID-19}

Como ya se mencionó, de la literatura histórica se desprende que la naturaleza de la pandemia, el tipo de instituciones y la distribución de los ingresos determinan si las élites aceptarán o rechazarán las iniciativas para aumentar la distribución de los recursos y establecer nuevas instituciones. Aún es demasiado prematuro determinar si los grupos de élite están dispuestos a transformar las instituciones en el contexto de la actual pandemia. Los datos del Observatorio COVID-19 en América Latina y el Caribe sobre las medidas económicas impulsadas por los gobiernos en respuesta al COVID-19 hasta septiembre de 2020 muestran que un tercio de dichas medidas han sido políticas orientadas a las empresas (véase el gráfico 1). El énfasis en políticas de ese tipo es particularmente acentuado en países con gobiernos que favorecen una agenda proempresarial, como el Brasil, Chile y Colombia.

\section{Gráfico 1}

América Latina (18 países): medidas económicas aplicadas para enfrentar la pandemia de COVID-19, por tipo de medida y país, marzo a septiembre de 2020

(En número)

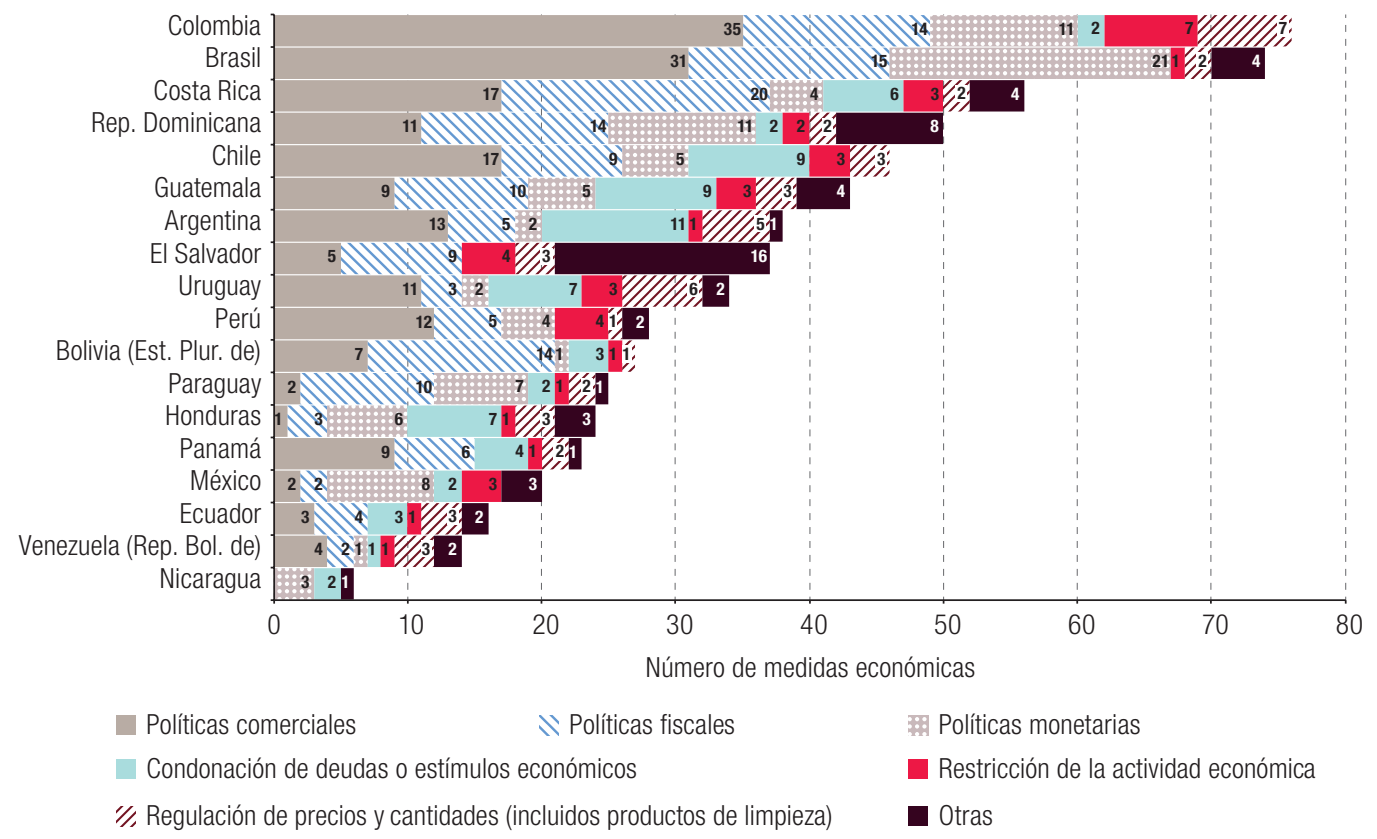

Fuente: Elaboración propia, sobre la base de Comisión Económica para América Latina y el Caribe (CEPAL), "Follow up of the evolution of COVID-19 Measures", 2020 [en línea] https://cepalstat-prod.cepal.org/forms/covid-countrysheet/index.html.

Sin embargo, el contenido de estas políticas, o la medida en que sean el resultado de la presión de las élites, probablemente varíen. En los siguientes párrafos, y sobre la base de los trabajos de Bull y Aguilar-Støen (2019), Cárdenas, Robles Rivera y Martínez-Vallejo (2020), Robles Rivera (2014), Sánchez-Ancochea y Puig (2013) y Segovia (2005), analizamos las reacciones de la élites durante la pandemia de COVID-19, haciendo especial hincapié en Centroamérica, una región donde las élites han tenido una clara influencia (véase el cuadro 3). Una comparación preliminar de la reacción de los grupos de élite a las políticas para enfrentar la pandemia sugiere la existencia de una elevada correlación entre las respuestas y el acceso de las élites al gobierno, además de su origen étnico 
(Pérez-Sáinz, 2016; Sánchez-Ancochea y Martínez-Franzoni, 2020). En Guatemala y en Honduras, las redes de élites y los grupos empresariales diversificados en manos de familias han desempeñado un papel crucial en la definición de la agenda política (Bull, Castellacci y Kasahara, 2014). En estos países, tradicionalmente las élites se han opuesto con vehemencia a las reformas fiscales y a cualquier iniciativa de expansión estatal, pese al reciente reconocimiento de la importancia de las instituciones del Estado (Bull y Aguilar-Støen, 2019). Como resultado, los niveles de tributación siguen siendo los más bajos en América Latina (OCDE y otros, 2020). Lejos de promover cambios a nivel institucional, durante la crisis del COVID-19 los gobiernos han recurrido a la filantropía empresarial para mitigar la falta de insumos médicos y la escasez de alimentos (Cárdenas, Robles Rivera y Martínez-Vallejo, 2020). Por ejemplo, en julio, el Consejo Hondureño de la Empresa Privada (COHEP) inauguró un centro de atención temprana y estabilización para pacientes con COVID-19 en asociación con el Municipio del Distrito Central y, en septiembre, se inauguró el Hospital de Santa Lucía Cotzumalguapa en Guatemala, con donaciones en especie (el sitio de la construcción y los equipos médicos) de la Asociación de Azucareros de Guatemala (ASAZGUA) (Mutz, 2020). De manera similar, en México, el magnate de negocios Carlos Slim se ofreció a financiar para el mercado mexicano la producción de la vacuna que están desarrollando la Universidad de Oxford y AstraZeneca (El Heraldo de México, 2020). Más que fortalecer las capacidades estatales, estas acciones, indudablemente urgentes y necesarias, han aumentado el poder de negociación de las élites.

Cuadro 3

Centroamérica (5 países): propuestas y medidas adoptadas por las élites en respuesta a la pandemia de COVID-19

\begin{tabular}{lll}
\hline País & Tipo de relación con el gobierno & Principales propuestas y medidas \\
\hline El Salvador & No participación & $\begin{array}{l}\text { Filantropía empresarial } \\
\text { Exenciones tributarias } \\
\text { Oposición al confinamiento }\end{array}$ \\
\hline Honduras & Captura & $\begin{array}{l}\text { Filantropía empresarial } \\
\text { Exenciones tributarias }\end{array}$ \\
& & Financiamiento extranjero \\
\hline Costa Rica & Cooperación & Exenciones tributarias \\
& & Reestructuración del sector de los empleos públicos \\
& Reducción de las contribuciones a la seguridad social \\
\hline Guatemala & Captura & Filantropía empresarial \\
& & Exenciones tributarias \\
& Financiamiento extranjero \\
\hline Nicaragua & No participación & Exenciones al impuesto sobre el valor agregado \\
& & Financiamiento extranjero \\
\hline
\end{tabular}

Fuente: Elaboración propia, sobre la base de Cárdenas, Robles Rivera y Martínez-Vallejo, "Élites empresariales y desigualdad en tiempos de pandemia en América Latina", Revista Española de Sociología, vol. 29, № 3, 2020.

Por otro lado, en los países vecinos de El Salvador y Nicaragua, la pandemia podría acelerar los actuales cambios a nivel de las élites, con resultados que aún son sumamente inciertos. El pacto de cogobierno entre la élite nicaragüense tradicional y la familia del presidente Ortega (Spalding, 2017) naufragó en 2018, cuando se recurrió a la represión masiva y a violaciones a los derechos humanos para responder a las manifestaciones contra una reforma del sistema jubilatorio. Aun durante la pandemia, las élites nicaragüenses han procurado establecer, sin éxito, nuevos canales de comunicación con la familia Ortega. Las reacciones de las élites a la pandemia en El Salvador han sido similares. Si bien tradicionalmente las élites salvadoreñas han desempeñado un papel político crucial (Robles Rivera, 2017; Segovia, 2005), el Presidente, Nayib Bukele, las ha enfrentado públicamente desde el comienzo de la crisis, lo que ha suscitado la indignación pública de las asociaciones empresariales contra las medidas de confinamiento impulsadas por el Gobierno (Cárdenas, Robles Rivera y Martínez-Vallejo, 2020). 
En Costa Rica, las reacciones de las élites han sido un reflejo de su acceso y su cooperación con el gobierno y el congreso de centro-derecha ${ }^{10}$. La administración de Alvarado designó a importantes miembros de la comunidad empresarial para supervisar el sector económico del país (Cárdenas, Robles Rivera y Martínez-Vallejo, 2020). Desde el mismo comienzo de la crisis del COVID-19, las élites han promovido activamente políticas y cambios mediante la asociación nacional de empresas, la Unión Costarricense de Cámaras y Asociaciones del Sector Empresarial Privado, y una influyente organización filantrópica, Horizonte Positivo. Han procurado establecer acuerdos para flexibilizar la reglamentación empresarial ("tramitología") (Murillo, 2020), y han bregado por la aplicación de medidas de austeridad y de reducción del tamaño del Estado, a la vez que han rechazado la creación de nuevos impuestos (UCCAEP, 2020). Esto parece indicar que, en los países donde están bien cohesionadas y ejercen el control sobre los recursos, las élites buscan supervisar a las autoridades públicas, no desafiarlas.

\section{Conclusiones}

Ha quedado relativamente claro que el COVID-19 no será el "gran igualador" que inicialmente se pensó que sería. La pandemia aumentará la pobreza y tendrá efectos particularmente profundos sobre los grupos de ingresos más bajos, no sobre las clases medias, por la diferente naturaleza de su trabajo. La gran cuestión ahora es cuáles serán los efectos futuros del COVID-19 sobre la desigualdad en materia de riqueza e ingresos.

América Latina se caracteriza por un elevado nivel de desigualdad en esos ámbitos. Si bien, entre 2003 y 2014, la distribución de los ingresos mejoró, no ocurrió lo mismo con la distribución de la riqueza. Además, los mecanismos que permitieron mejorar la distribución de los ingresos resultaron ser insostenibles, en parte por motivos económicos, pero principalmente por causas políticas. Las estrategias utilizadas no lograron producir efectos positivos de retroalimentación ni propiciar reformas a largo plazo y, en algunos casos, suscitaron la férrea oposición de las élites (Chiasson-LeBel y Larrabure, 2019).

La literatura histórica parece indicar que las pandemias pueden generar las condiciones necesarias para mejorar la distribución si se transforman en una "coyuntura crítica" que obligue a las élites a pensar en la inclusión y la distribución de los recursos. En este trabajo, se analizó la concentración de los recursos y las reacciones de las élites a las diferentes medidas de redistribución adoptadas en respuesta a la pandemia de COVID-19. Los datos preliminares sugieren que, de hecho, durante la pandemia se ha producido una transferencia de los recursos hacia los estratos más altos de la sociedad. No obstante, no todos los grupos de élites se han beneficiado de la misma forma. Las tendencias señalan que, a raíz del COVID-19, el ingreso se ha concentrado en manos de los superricos (multimillonarios), y en particular, si bien hay excepciones, en quienes pertenecen a los sectores tecnológicos.

Hay pocos indicios de que los individuos muy acaudalados hayan encontrado en la pandemia un motivo para apoyar las reformas distributivas. Por el contrario, su participación en varias iniciativas de filantropía empresarial podría, a la larga, perpetuar el statu quo. El avance de la robotización y otros fenómenos también reducen las probabilidades de que, aun en un contexto de escasez de mano de obra, algo que históricamente sucede tras las pandemias, las reivindicaciones por aumentos salariales tengan respuesta.

La pandemia de COVID-19 está lejos de haber terminado. Podría generar una conmoción tan profunda que suscite cambios más trascendentales en algunos países; en particular, en aquellos que ya estaban atravesando graves convulsiones, como Chile. Los lugares donde podrían materializarse

\footnotetext{
${ }^{10}$ Según datos del Proyecto de Élites Parlamentarias en América Latina (PELA, 2018), en general, y por su posicionamiento ideológico, los partidos políticos elegidos para integrar el congreso en el período 2018-2022 pueden describirse como de centro-derecha.
} 
conmociones de ese tipo también dependerán de los cambios en el contexto mundial. Está claro que estamos siendo testigos de una concentración de los recursos mundiales en manos de dos grupos de actores: las empresas multinacionales más importantes (la mayoría de ellas de procedencia estadounidense), y las empresas chinas. El tipo de vínculos que dichas empresas establezcan con las élites locales de América Latina será un factor fundamental en lo referido a su futuro, el de las instituciones y el de la redistribución.

\section{Bibliografía}

Acemoglu, D. y J. Robinson (2020), El pasillo estrecho: estados, sociedades y cómo alcanzar la libertad, Barcelona, Ediciones Deusto.

Alvaredo, F. y otros (eds.) (2018), World Inequality Report 2018, Cambridge, The Belknap Press of Harvard University Press.

Amarante, V. y J. Jiménez (2015), "Desigualdad, concentración y rentas altas en América Latina", Desigualdad, concentración del ingreso y tributación sobre las altas rentas en América Latina, J. Jiménez (ed.), Libros de la CEPAL, № 134 (LC/G.2638-P), Santiago, Comisión Económica para América Latina y el Caribe (CEPAL).

Aspachs, O. y otros (2020), "Real-time inequality and the welfare State in motion: evidence from COVID-19 in Spain", CEPR Discussion Papers, № DP15118, Londres, Centro de Investigación en Economía y Política.

Banco Mundial (2018a), "Índice de Gini" [en línea] https://datos.bancomundial.org/indicador/SI.POV.GINI [fecha de consulta: 21 de enero de 2020].

— (2018b), "Participación en el ingreso del 10\% mejor remunerado de la población” [en línea] https://datos. bancomundial.org/indicador/SI.DST.10TH.10 [fecha de consulta: 21 de enero de 2020].

Béland, L. P., A. Brodeur y T. Wright (2020), "The short-term economic consequences of COVID-19: exposure to disease, remote work and government response", IZA Discussion Papers, № 13159, Bonn, Institute of Labor Economics (IZA).

Bernal, T. y F. Michel (2016), "Diseño de un Assessment Center, buscando productividad de los recursos humanos en los procesos productivos de las pequeñas y medianas empresas", tesis de maestría, Guayaquil, Universidad de Guayaquil [en línea] http://repositorio.ug.edu.ec/handle/redug/41551.

Bielschowsky, R. y M. Torres (comps.) (2018), Desarrollo e igualdad: el pensamiento de la CEPAL en su séptimo decenio. Textos seleccionados del período 2008-2018, Colección 70 años, № 1 (LC/PUB.2018/7-P), Santiago, Comisión Económica para América Latina y el Caribe (CEPAL).

Bull, B. (2020), "Venezuela y el capitalismo del Siglo XXI: cambios visibles", La Lettre de l'IHEAL-CREDA, $N^{\circ} 40$, abril.

(2019), "Combating inequality: is there a 'Scandinavian way' to reduce inequality in Latin America?", Iberoamericana - Revista Nórdica de Estudios de Latinoamérica y el Caribe, vol. 48, № 1.

(2014), "Towards a political economy of weak institutions and strong elites in Central America", ERLACS - Revista Europea de Estudios Latinoamericanos y del Caribe, № 97.

Bull, B. y M. Aguilar-Støen (2019), "Peace-building and business elites in Guatemala and El Salvador: explaining the discursive 'institutional turn'”, Conflict, Security \& Development, vol. 19, № 1.

Bull, B., F. Castellacci e Y. Kasahara (2014), Business Groups and Transnational Capitalism in Central America: Economic and Political Strategies, Basingstoke, Palgrave Macmillan.

Busso, M. y J. Messina (eds.) (2020), La crisis de la desigualdad: América Latina y el Caribe en la encrucijada, Banco Interamericano de Desarrollo (BID) [en línea] https://doi.org/10.18235/0002629.

Capgemini (2020), "World Wealth Report 2020" [en línea] https://worldwealthreport.com/.

Cárdenas, J. y F. Robles Rivera, F. (2020), Business Elites' Sources of Power and State Capture in Panama, Ginebra, Instituto de Investigaciones de las Naciones Unidas para el Desarrollo Social (UNRISD), en prensa.

Cárdenas, J., F. Robles Rivera y D. Martínez-Vallejo (2020), "Élites empresariales y desigualdad en tiempos de pandemia en América Latina", Revista Española de Sociología, vol. 29, № 3.

Castañeda, N. (2018), "Business groups, tax efficiency, and regressivity in Colombia", Rethinking taxation in Latin America, J. Atria, C. Groll y M. Valdés (eds.), Cham, Springer International Publishing.

CEPAL (Comisión Económica para América Latina y el Caribe) (2020a), "El desafío social en tiempos del COVID-19", Informe Especial COVID-19, № 3, Santiago, 12 de mayo. (2020b), "Mipymes y el COVID-19" [en línea] https://www.cepal.org/en/node/51732. 
- (2020c), "Medidas de recuperación del sector turístico en América Latina y el Caribe: una oportunidad para promover la sostenibilidad y la resiliencia", Informes COVID-19, julio. (2019), Panorama Fiscal de América y el Caribe, 2019 (LC/PUB.2019/8-P), Santiago.

Chiasson-LeBel, T. y M. Larrabure (2019), "Latin America's changing balance of class forces: an introduction", ERLACS - Revista Europea de Estudios Latinoamericanos y del Caribe, № 108.

Coffey, C. y otros (2020), "Tiempo para el cuidado: el trabajo de cuidados y la crisis global de desigualdad", Informe de Oxfam, Oxford, Oxfam, 20 de enero [en línea] https://www.oxfam.org/es/informes/tiempopara-el-cuidado.

Cohn, S. y G. Alfani (2007), "Households and plague in early modern Italy", The Journal of Interdisciplinary History, vol. 38, № 2.

Collier, R. y D. Collier (1991), Shaping the Political Arena: Critical Junctures, the Labor Movement, and Regime Dynamics in Latin America, Princeton, Princeton University Press.

Cornia, G. (2014), Falling Inequality in Latin America: Policy Changes and Lessons, Oxford, Oxford University Press.

De Haan, J. y J. E. Sturm (2017), "Finance and income inequality: a review and new evidence", European Journal of Political Economy, vol. 50, diciembre.

De Jesús, L. (2020), "Microestados liderados por bandas criminales: crece la extorsión en Venezuela en medio de la pandemia", El Nacional, 31 de agosto [en línea] https://www.elnacional.com/venezuela/ microestados-liderados-por-bandas-criminales-crece-la-extorsion-en-venezuela/.

Delaporte, I. y W. Peña (2020), "Working from home under covid-19: who is affected? Evidence from Latin American and Caribbean countries", COVID Economics, Vetted and Real-Time Papers, № 14, Londres, Centro de Investigación en Economía y Política, 6 de mayo.

Durand, F. (2019), La captura del Estado en América Latina: reflexiones teóricas, Lima, Pontificia Universidad Católica del Perú (PUCP), Fondo Editorial.

El Heraldo de México (2020), "Así informó Carlos Slim a AMLO sobre proyecto para crear vacuna contra Covid-19", 13 de agosto [en línea] https://heraldodemexico.com.mx/pais/asi-informo-carlos-slim-a-amlosobre-proyecto-para-crear-vacuna-contra-covid-19/.

Euraque, D. (2019), "La configuración histórica de las élites de Honduras ante el golpe de Estado del 2009", Anuario de Estudios Centroamericanos, vol. 45, San José, Universidad de Costa Rica (UCR).

Faguet, J. P., F. Sánchez y M. J. Villaveces (2020), "The perversion of public land distribution by landed elites: power, inequality and development in Colombia", World Development, vol. 136.

Fairfield, T. (2015), Private Wealth and Public Revenue in Latin America: Business Power and Tax Politics, Nueva York, Cambridge University Press.

Fukuyama, F. (2016), Los orígenes del orden político: desde la prehistoria hasta la Revolución francesa, Barcelona, Ediciones Deusto.

Furceri, D. y otros (2020), "Will Covid-19 affect inequality? Evidence from past pandemics", Covid Economics, Vetted and Real-Time Papers, № 12, Londres, Centro de Investigación en Economía y Política, 1 de mayo.

Galasso, V. (2020), "Covid: not a great equaliser", Covid Economics, Vetted and Real-Time Papers, № 19, Londres, Centro de Investigación en Economía y Política, 18 de mayo.

Giordano, P. (coord.) (2020), "Estimaciones de las tendencias comerciales: América Latina y el Caribe, edición 2020, actualización 1T”, Banco Interamericano de Desarrollo (BID) [en línea] https://doi.org/10.18235/0002390.

Gneiting, U., N. Lusiani e I. Tamir (2020), "Power, profits and the pandemic: from corporate extraction for the few to an economy that works for all", Informe de Oxfam, Oxford, Oxfam, 10 de septiembre [en línea] https://oxfamilibrary.openrepository.com/handle/10546/621044.

Karlsson, M., T. Nilsson y S. Pichler (2014), "The impact of the 1918 Spanish flu epidemic on economic performance in Sweden: an investigation into the consequences of an extraordinary mortality shock", Journal of Health Economics, vol. 36, julio.

Kasahara, Y. (2012), "Should I stay or should I go? A comparative study of banking sector policies and the strategies of Central American business groups", Business and Politics, vol. 14, № 4.

Kitchener, S. (2020), "Latin American Equity Markets Staged Recovery in Q2 2020 despite Continuing Battle with COVID-19", S\&P Global, 21 de julio [en línea] https://www.spglobal.com/en/research-insights/articles/ latin-american-equity-markets-staged-recovery-in-q2-2020-despite-continuing-battle-with-covid-19.

López Vega, R., E. Figueroa y C. Gutiérrez (2013), “La ‘parte del león’: nuevas estimaciones de la participación de los súper ricos en el ingreso de Chile", Documentos de Trabajo, №379, Santiago, Universidad de Chile.

Loureiro, P. (2020), "Class inequality and capital accumulation in Brazil, 1992-2013", Cambridge Journal of Economics, vol. 44, № 1, enero. 
Mamelund, S. (2017), "Profiling a pandemic: who were the victims of the Spanish flu?", Natural History, número especial, septiembre [en línea] https://oda-hioa.archive.knowledgearc.net/handle/10642/5480.

Mari, A. (2019), "Digital economy will represent over half of Latin America's GDP by 2022", ZDNet, 22 de marzo [en línea] https://www.zdnet.com/article/digital-economy-will-represent-over-half-of-latin-americasgdp-by-2022/.

Mein, S. (2020), "COVID-19 and health disparities: the reality of 'the great equalizer'", Journal of General Internal Medicine, vol. 35, № 8, agosto.

Moene, K. (2015), "The social upper class under social democracy", Nordic Economic Policy Review, № 2, Copenhague, Consejo Nórdico de Ministros.

Morgan, M. (2017), "Extreme and persistent inequality: new evidence for Brazil combining national accounts, surveys and fiscal data, 2001-2015", WID.World Working Paper series, № 12 [en línea] https:// fernandonogueiracosta.files.wordpress.com/2017/09/extreme-and-persistent-inequality-new-evidencefor-brazil-marc-morgan-wid-wp-2017-12.pdf.

Mungaray Lagarda, A. y otros (2015), "Emprendimientos de micro y pequeñas empresas mexicanas en un escenario local de crisis económica: el caso de Baja California, 2008-2011”, Frontera Norte, vol. 27, Nㅜ 53.

Murillo, A. (2020), "Asociación que presidió el ministro Garnier intervendrá en reducir trámites de Gobierno", Semanario Universidad, 28 de mayo [en línea] https://semanariouniversidad.com/pais/asociacion-quepresidio-el-ministro-garnier-intervendra-en-reducir-tramites-de-gobierno/.

Mutz, V. (2020), "Inauguran hospital permanente en Santa Lucía Cotzumalguapa", República, 2 de septiembre [en línea] https://republica.gt/2020/09/02/inauguran-hospital-permanente-en-santa-lucia-cotzumalguapa/.

Noe-Bustamante, L. (2020), "Amid COVID-19, remittances to some Latin American nations fell sharply in April, then rebounded", Pew Research Center, 31 de agosto [en línea] https://www.pewresearch.org/ fact-tank/2020/08/31/amid-covid-19-remittances-to-some-latin-american-nations-fell-sharply-in-aprilthen-rebounded/.

OCDE (Organización de Cooperación y Desarrollo Económicos)/Facebook (2020), "Global State of Small Business Report: Wave II Update" [en línea] https://dataforgood.fb.com/wp-content/uploads/2020/08/ State-of-Small-Business-Report-Wave-II-Summary.pdf.

OCDE (Organización de Cooperación y Desarrollo Económicos) y otros (2020), Estadísticas tributarias en América Latina y el Caribe 2020, París.

OIT (Organización Internacional del Trabajo) (2020a), "OIT: fuerte aumento del desempleo en América Latina y el Caribe deja a millones sin ingresos", 1 de julio [en línea] https://www.ilo.org/americas/sala-de-prensa/ WCMS_749663/lang--es/index.htm.

(2020b), Observatorio de la OIT: la COVID-19 y el mundo del trabajo, sexta edición, 23 de septiembre [en línea] https://www.ilo.org/wcmsp5/groups/public/@dgreports/@dcomm/documents/briefingnote/ wcms_755917.pdf.

Oxfam (2020), "Aumentan los milmillonarios de América Latina a medida que la región más desigual del mundo se hunde bajo el impacto del coronavirus", 27 de julio [en línea] https://www.oxfam.org/es/notas-prensa/ aumentan-los-mil-millonarios-de-america-latina-medida-que-la-region-mas-desigual.

Peacock, A. y J. Wiseman (1961), The Growth of Public Expenditure in the United Kingdom, Princeton, Princeton University Press [en línea] https://www.nber.org/books/peac61-1.

PELA (Elites Parlamentarias de América Latina) (2018), "Bases de datos países" [en línea] https://oir.org.es/ pela/bases-datos-paises/.

Pérez, O. (2011), Political Culture in Panama: Democracy after Invasion, Nueva York, Palgrave Macmillan.

Pérez-Sáinz, J. (2016), Una historia de la desigualdad en América Latina: la barbarie de los mercados, desde el siglo XIX hasta hoy, Buenos Aires, Siglo XXI Editores.

Piketty, T. (2014), El capital en el siglo XXI, Ciudad de México, Fondo de Cultura Económica.

Robles Rivera, F. (2017), "Élites en El Salvador: cambios y continuidades (2000-2016)", Anuario de Estudios Centroamericanos, vol. 43, San José, Universidad de Costa Rica (UCR). (2014), "Transformaciones y concentración en grupos de poder económico en Costa Rica (1980-2012)", Revista Mexicana de Sociología, vol. 76, № 1.

Rocha, J. (2008), "12 mil millones en remesas: ¿Quién se come ese mandado?”, Revista Envío, №313, Managua, Universidad Centroamericana, abril.

Rodríguez Weber, J. (2018), "Alta desigualdad en América Latina: desde cuándo y por qué", Munich Personal RePEC Archive, № 87619, junio.

Rovira-Kaltwasser, C. (2018), "Political elites in Latin America", The Palgrave Handbook of Political Elites, H. Best y J. Highley (eds.), Londres, Macmillan Publishers. 
Ruiz, S. (2020), “¿Quién paga la cuenta? Gravar la riqueza para enfrentar la crisis de la COVID-19 en América Latina y el Caribe", Nota Informativa de Oxfam, 27 de julio [en línea] https://oxfamilibrary.openrepository. com/handle/10546/621033.

Rutter, P. y otros (2012), "Socio-economic disparities in mortality due to pandemic influenza in England", International Journal of Public Health, vol. 57, № 4.

Sánchez-Ancochea, D. y J. Martínez-Franzoni (2020), Pactos sociales al servicio del bienestar en América Latina: ¿qué son y cuál es su papel en tiempos de crisis?, en prensa.

Sánchez-Ancochea, D. y S. Puig (eds.) (2013), Handbook of Central American Governance, Abingdon, Routledge.

Santos, H. y G. Fraga (2020), "Corrupción, estructura productiva y desarrollo económico en los países en desarrollo", Revista CEPAL, № 130 (LC/PUB.2020/4-P), Santiago, Comisión Económica para América Latina y el Caribe (CEPAL).

Scheidel, W. (2018), El gran nivelador: violencia e historia de la desigualdad desde la Edad de Piedra hasta el siglo XXI, Barcelona, Editorial Crítica.

Scheve, K. y D. Stasavage (2017), "Wealth inequality and democracy", Annual Review of Political Science, vol. 20, mayo.

Segovia, A. (2005), Integración real y grupos de poder económico en América Central: implicaciones para el desarrollo y la democracia de la región, San José, Fundación Friedrich Ebert (FESUR).

Simonsen, L. y otros (2013), "Global mortality estimates for the 2009 influenza pandemic from the GLaMOR project: a modeling study", PLOS Medicine, vol. 10, N 11.

Sokol, M. y L. Pataccini (2020), "Winners and losers in coronavirus times: financialisation, financial chains and emerging economic geographies of the COVID-19 pandemic", Journal of Economic and Social Geography, vol. 111, № 3.

Solimano, A. (2015), Elites económicas, crisis y el capitalismo del siglo XXI: la alternativa de la democracia económica, Santiago, Fondo de Cultura Económica.

Sosa Iglesias, E. (2017), "Transformaciones en las élites económicas, estado y el proceso de democratización y desdemocratización: el caso de Honduras, 1990-2017", Anuario de Estudios Centroamericanos, vol. 43, San José, Universidad de Costa Rica (UCR).

Spalding, R. (2017), "Los empresarios y el estado posrevolucionario: el reordenamiento de las élites y la nueva estrategia de colaboración en Nicaragua", Anuario de Estudios Centroamericanos, vol. 43, San José, Universidad de Costa Rica (UCR).

Starr, P. (2019), Entrenchment: Wealth, Power, and the Constitution of Democratic Societies, New Haven, Yale University Press.

Trejos, J. y L. Oviedo (2012), "Cambios en la distribución del ingreso familiar en Costa Rica durante la primera década del siglo XXI", Revista de Ciencias Económicas, vol. 30, № 2, San José, Universidad de Costa Rica (UCR).

Turchin, P. (2018), Historical Dynamics: Why States Rise and Fall, Princeton, Princeton University Press.

UCCAEP (Unión Costarricense de Cámaras y Asociaciones del Sector Empresarial Privado) (2020), "Un pacto por el empleo: Día Nacional de la Empresa Privada", San José, 25 de febrero [en línea] https:// www.uccaep.or.cr/images/comunicados/Pacto-por-el-empleo-UCCAEP.pdf.

Ventrici, P., D. Krepki y H. Palermo (2020), "Sector software y la situación respecto de la pandemia de COVID-19", El trabajo en los tiempos del COVID-19, № 2, Buenos Aires, Centro de Estudios e Investigaciones Laborales (CEIL), 8 de junio. 